\title{
Echocardiographic Predictors of the Need for Infundibular Wedge Resection in Infants with Aortic Arch Obstruction, Ventricular Septal Defect and Subaortic Stenosis
}

\author{
L. LuAnn Minich, MD, A. Rebecca Snider, MD, Edward L. Bove, MD, and Flavian M. Lupinetti, MD
}

nfants with aortic arch obstruction and outlet ventricular septal defect can have posterior displacement of the infundibular septum into the left ventricular outflow tract causing varying degrees of subaortic stenosis. ${ }^{1-3}$ Because of the large ventricular septal defect, left ventricular outflow tract velocities are frequently normal. For this reason, Doppler peak gradients are often not helpful for assessing the severity of the outflow tract narrowing preoperatively. Preoperative evaluation of the degree of subaortic obstruction and, thus, the need for surgical intervention is usually based on qualitative assessment of the anatomic 2-dimensional echocardiographic image. ${ }^{2-6}$ This study defines 2-dimensional echocardiographic predictors of the need for subaortic resection in infants with aortic arch obstruction, outlet ventricular septal defect and posterior deviation of the infundibular septum.

From November 1987 to November 1991, 12 consecutive infants with this combination of cardiac defects were evaluated with 2-dimensional and Doppler echocardiography. Ten of the 12 patients had no previous cardiac evaluation, whereas 2 patients had undergone prior surgery at a referring institution - 1 for repair of an interrupted aortic arch and patch closure of the ventricular septal defect and the other for repair of an interrupted aortic arch alone. The patients ranged in age from 2 days to 9 months (mean 42 days) and in weight from 1.3 to $7.1 \mathrm{~kg}$ (mean 3.5). The initial diagnosis was interrupted aortic arch in 6 infants and coarctation of the aorta in 6 infants. Associated defects included an atrial septal defect in 10 patients, patent ductus arteriosus in 6 , bicuspid aortic valve in 5 , mild mitral regurgitation in 3 , and anomalous right subclavian artery in 1. Of the 12 patients, 11 underwent patch closure of the ventricular septal defect by a transtricuspid approach, 10 had primary aortic arch repair, 9 had wedge resection of the infundibular septum ( 8 through the tricuspid valve and ventricular septal defect and 1 through a transaortic approach), and 1 had revision of a previous aortic arch repair.

The patients' echocardiographic measurements were compared with those of a control group of age and weight-matched infants with normal hearts by 2-dimensional and Doppler echocardiography. The control group consisted of 15 infants whose ages ranged from I day to 2 months (mean 13 days) and whose weights ranged from 2 to $4 \mathrm{~kg}$ (mean 3.1). In these infants, echocardiographic evaluation was performed because of noncardiac congenital anomalies in 9, murmur in 5, and arrhythmia in 1.

The following measurements were obtained from the 2-dimensional and Doppler echocardiograms. From the

From the Departments of Pediatrics and Surgery, C.S. Mott Children's Hospital, University of Michigan Medical Center, Ann Arbor, Michigan. Manuscript received and accepted June 25, 1992. parasternal long-axis view, the diameter of the left ventricular outflow tract was measured using on-line electronic calipers (Figure 1). Measurements were made at end-systole and end-diastole from inner edge to inner edge.

In a similar fashion, the diameter of the descending aorta was measured at end-systole and end-diastole at the level of the diaphragm from the subcostal sagittal view. To allow comparison of different-sized hearts, the diameter of the left ventricular outflow tract was then divided by the diameter of the descending aorta at the diaphragm. Left ventricular outflow tract gradients were calculated with Doppler echocardiography using the Bernoulli equation and peak velocities measured with continuous-wave Doppler from the apical view. Echocardiographic examinations were performed both preand postoperatively in all patients.

For all measurements, $\geq 3$ cardiac cycles were analyzed and the results were averaged. The patient and control groups were compared using unpaired Student's $\mathrm{t}$ tests. A p value $<0.01$ was used to indicate a significant intergroup difference. The values reported here are mean \pm standard deviation.

The patient and the control group did not differ in age or weight. Compared with control infants, the patients had smaller left ventricular outflow tract diameters in systole and diastole, similar descending aorta diameters in systole and diastole, and smaller left ventricular outflow tract/descending aorta ratios (Figure 2). Because of cardiac motion and ventricular contraction, left ventricular outflow tract diameters could be measured with greater ease and certainty in diastole than in systole. In normal infants, the left ventricular outflow tract/descending aorta diastolic ratio was $1.3 \pm 0.14$.

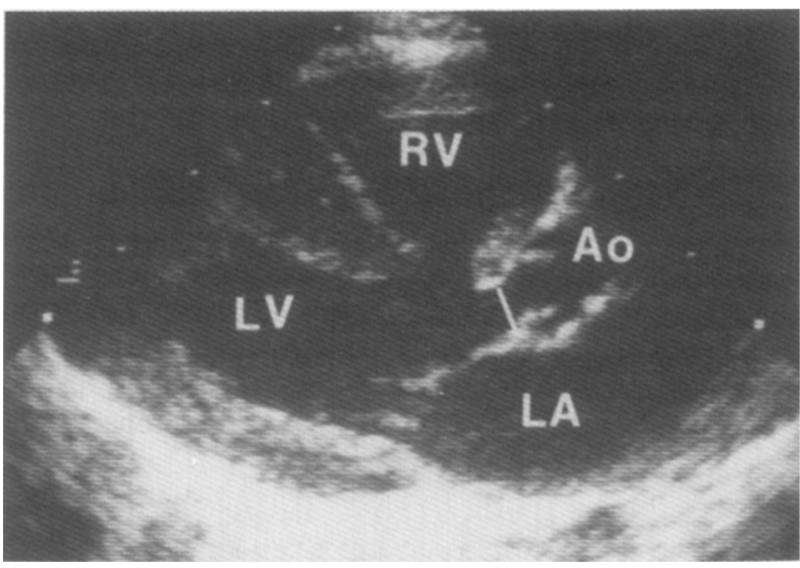

FIGURE 1. Parastemal long-axis view of the left ventricular outfiow tract with calpers demonstrating the outilow tract diameter. Note the posterior deviation of the infundibular septum. Ao = aorta; LA = left atrium; $\mathbf{L V}=$ left ventricle; RV = right ventricle. 
FIGURE 2. Comparisons of the preoperative echocardiographic results. DAO = descending aorta; LVOT = left ventricular outflow tract.

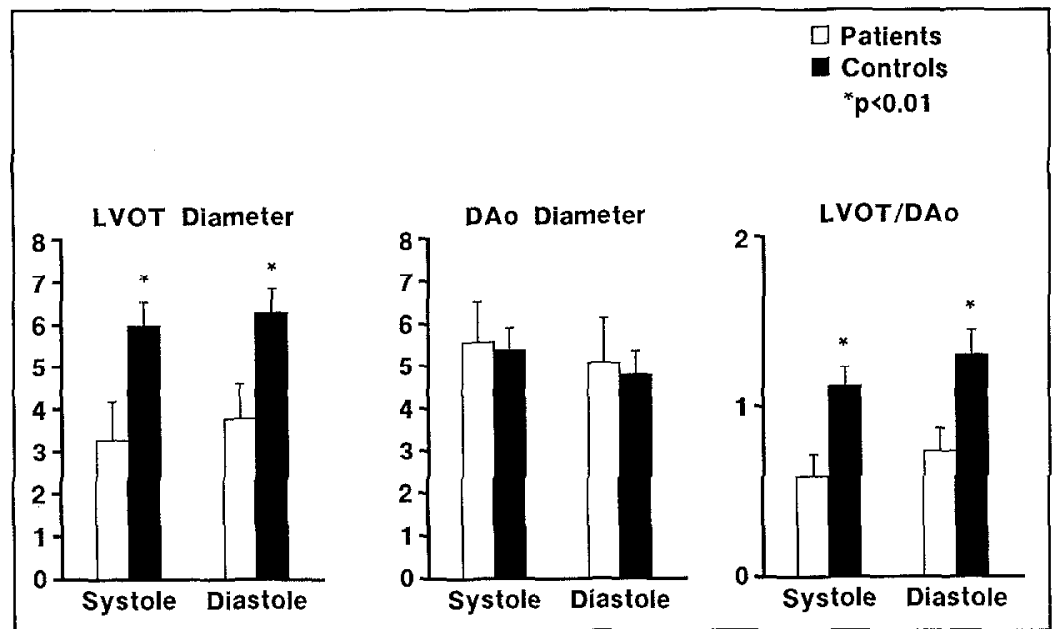

On the most recent follow-up Doppler examination obtained from 12 days to 11 months after operation (median 24 days), the left ventricular outflow tract peak gradient ranged from 0 to $36 \mathrm{~mm} \mathrm{Hg}$ (mean 15).

The preoperative left ventricular outflow tract/descending aorta ratio in diastole was $>1.0$ in only $1 \mathrm{pa}$ tient and this patient required no subaortic resection and has no residual gradient. The preoperative left ventricular outflow tract/descending aorta ratio in diastole was $<1.0$ in 11 patients. Nine of these 11 patients with ratios of 0.55 to 0.92 had subaortic resections and had no significant left ventricular outflow tract gradient on the postoperative echocardiographic examination $(10 \mathrm{~mm}$ $\mathrm{Hg}$ ). Two of the 11 patients with left ventricular outflow tract/descending aorta ratios $<1.0$ (mean 0.73 and 0.89 ) had no subaortic resection and now have the highest residual gradients postoperatively ( 30 and $36 \mathrm{~mm} \mathrm{Hg}$ ).

In this study, the ratio of the diameter of the left ventricular outflow tract to the diameter of the descending aorta was measured from 2-dimensional echocardiography in both systole and diastole in normal infants and in infants with aortic arch obstruction, ventricular septal defect and posterior deviation of the infundibulum. Because the diameter of the descending aorta at the diaphragm was similar in systole and diastole for both patient and control groups, this dimension was used to provide an index for comparisons of different-sized hearts. Because the left ventricular outflow tract was measured more easily in diastole, the diastolic left ventricular outflow tract/descending aorta ratio of the patient group was compared with the same ratio of the control group. Only 1 infant in the patient group had a diastolic left ventricular outflow tract/descending aorta ratio in the normal range $(>1.0)$. This infant had no subaortic resection and has not developed subaortic stenosis. Of the remaining 11 patients with diastolic ratios of $<1.0$, those who had no surgical resection of the posterior infundibulum have developed subaortic stenosis, whereas those who had surgical resection have not developed significant gradients. This short-term follow-up suggests that patients with a diastolic left ventricular outflow tract/descending aorta ratio of $<1.0$ are at risk of developing postoperative subaortic stenosis and therefore should undergo subaortic resection. Patients with a diastolic left ventricular outflow tract/descending aorta ratio of $>1.0$ do not appear to develop subaortic stenosis and therefore require no surgical intervention. Because this study is limited by small numbers and only short-term follow-up, continued investigation with a larger patient group and longer follow-up is warranted.

Thus, in normal infants, the left ventricular outflow tract/descending aorta diastolic ratio is $>1.0$. In infants with severe muscular subaortic stenosis caused by posterior deviation of the infundibular septum, an echocardiographic left ventricular outflow tract/descending aorta diastolic ratio of $<1.0$ suggests that wedge resection of the infundibular septum should be performed to provide adequate relief of the obstruction. We recommend that this ratio be included as part of the preoperative evaluation of all infants with aortic arch obstruction, outlet ventricular septal defect and posterior deviation of the infundibulum.

1. Freedom RM, Dischi R, Rowe RD. Pathologic anatomy of subaortic stenosis and atresia the first year of life. Am J Cardiol 1977;39:1035-1044.

2. Smallhorn JF, Anderson RH, Macartney FJ. Morphological characterization of ventricular septal defects associated with coarctation of aorta by cross-sectional echocardiography. Br Heart $J$ 1983;49:485-494.

3. Anderson RH, Lenox CC, Zuberbuhler JR. Morphology of ventricular septal defect associated with coarctation of aorta. Br Heart $J$ 1983;50:176-181.

4. Yasui $H$, Kado $H$, Nakano $E$, Yonenaga $K$, Mitani A, Tomita $Y$, Iwao $H$, Yoshii K, Mizoguchi Y, Sunagawa H. Primary repair of interrupted aortic arch and severe aortic stenosis in neonates. $J$ Thorac Cardiovasc Surg 1987;93: 539-545.

5. Ilbawi MN, Idriss FS, DeLeon SY, Muster AJ, Benson DW, Paul MH. Surgical management of patients with interrupted aortic arch and severe subaortic stenosis. Ann Thorac Surg 1988;45:174-180.

6. Iwahara M, Ino T, Nishimoto K, Park I, Akimoto K, Shimazaki S, Yabuta K, Tanaka A, Hosoda Y. Clinical features of aortic arch anomaly with malalignment ventricular septal defect. Ann Thorac Surg 1989;48:693-696. 